\title{
High resolution TEM imaging and analysis of the core-shell assembly of ferritin- poly 4-vinyl pyridine (P4VP)
}

\author{
Laying $\mathrm{Wu}^{*}{ }_{\dagger}$, Tao Li†, Douglas Blom*, Jibin Zhao*, Soumitra Ghoshroy*, Qian Wang ${ }_{\dagger}$ \\ *Electron Microscopy Center, University of South Carolina, 715 Sumter St., Columbia, SC 29208 \\ $\dagger$ Department of Chemistry and Biochemistry and Nanocenter, University of South Carolina, 631 \\ umter St., Columbia, SC 29208
}

The assembly between protein cages and polymers has potential to develop multifunctional materials with a wide range of applications like drug delivery, gene therapy, and tissue engineering $(1,2)$. In this study we presented the self-assembly between ferritin and poly 4-vinyl pyridine (P4VP) and subsequent formation of the core-shell structures. FESEM and high resolution TEM (HRTEM) and associated energy dispersive x-ray spectroscopy (EDS) techniques were utilized for visualization and characterization of the core-shell structures of ferritin-P4VP. Following the published protocol (3), a solution of P4VP (Mw 60,000 Da) in DMF $(2.0 \mathrm{mg} / \mathrm{mL}, 0.5 \mathrm{~mL})$ was slowly added into a solution of horse spleen ferritin (from Sigma-Aldrich) in pure water $(6.67 \mu \mathrm{g} / \mathrm{mL}, 16.5 \mathrm{~mL})$ under stirring for 30-45 minutes. Then the samples were dialyzed against pure water $(2 \times 1 \mathrm{~L})$ with the MW 3,500 cutoff dialysis tube (from Pierce). The final product analyzed by TEM (Hitachi H8000) and FESEM (Hitachi S4800) (Figure 1), showed typical core-shell like structures. To further confirm the location of ferritin, TEM tomography was used to analyze the ferritin-P4VP particles. Beginning with a conventional TEM cross section method, the structure of ferritin-P4VP was investigated in several ways. The optimal cross section conditions for such colloidal particles were developed under GTA fixation + acetone/resin infiltration after following step by step conditions described here: (1) glutaraldehyde (GTA) fixation + osmium tetraoxide $\left(\mathrm{OsO}_{4}\right)$ post fixation + ethanol $(\mathrm{EtOH})$ dehydration + acetone/resin (Spurr's or Micro-bed's) infiltration (A/R) + resin embedding (Emb) (Fig 2a), (2) GTA + $\mathrm{OsO}_{4}+\mathrm{EtOH}+\mathrm{Emb}$ (Fig 2b), (3) GTA + OsO4 $+\mathrm{A} / \mathrm{R}+\mathrm{Emb}$ (Fig 2c), (4) GTA $+\mathrm{EtOH}+\mathrm{Emb}($ Fig 2d), (5) GTA + Emb (Fig 2e), and (6) GTA + A/R + Emb (Fig 2f). Due to the iron oxide core, ferritin particles should appear as dark dots under electron microscope. Using condition 1, 2, or 3, the specimen turned dark as shown in Figure 2a-c. Clearly, it was not the ideal protocol to localize the ferritin particles of the core-shell structure. This phenomenon was caused by the binding of $\mathrm{OsO}_{4}$ to $\mathrm{P} 4 \mathrm{VP}$ which led the polymer component to become electron-dense. As the P4VP dissolved in ethanol, the whole core-shell structure of ferritin-P4VP became distorted and oriented during curing (Figure 2d). By elimination of acetone/rein infiltration step, a minor structural deformation was noted in the specimen (Fig 2e). In light of these observations, an optimal condition was achieved under condition 6 as shown in Figure 2f. Here the cross section of ferritin-P4VP showed a dark ring structure and these rings were most likely from ferritins. To prove the assumption, HRTEM was employed and the images revealed a large number of tiny dark particles with the size of one or two ferritins (Fig 3). Subsequent elemental analysis of the dark particles indicated the presence of elements $\mathrm{Fe}$ and $\mathrm{P}$ which constitute ferritin proteins. This study demonstrated that EM is a unique technique to examine the detailed structure of nanoassembly of protein cages and polymers. Our study will be critical for further development of bio/polymer coreshell materials for biomedical applications.

References (1) J. L. Doux et al., Human Gene Therapy, 12 (2001) 1611. (2) X. Zhang et al., Gene Delivery for Tissue Engineering, 58(4) (2006) 515. (3) T. Li et al., Small, 4(10) (2008) 1624. 

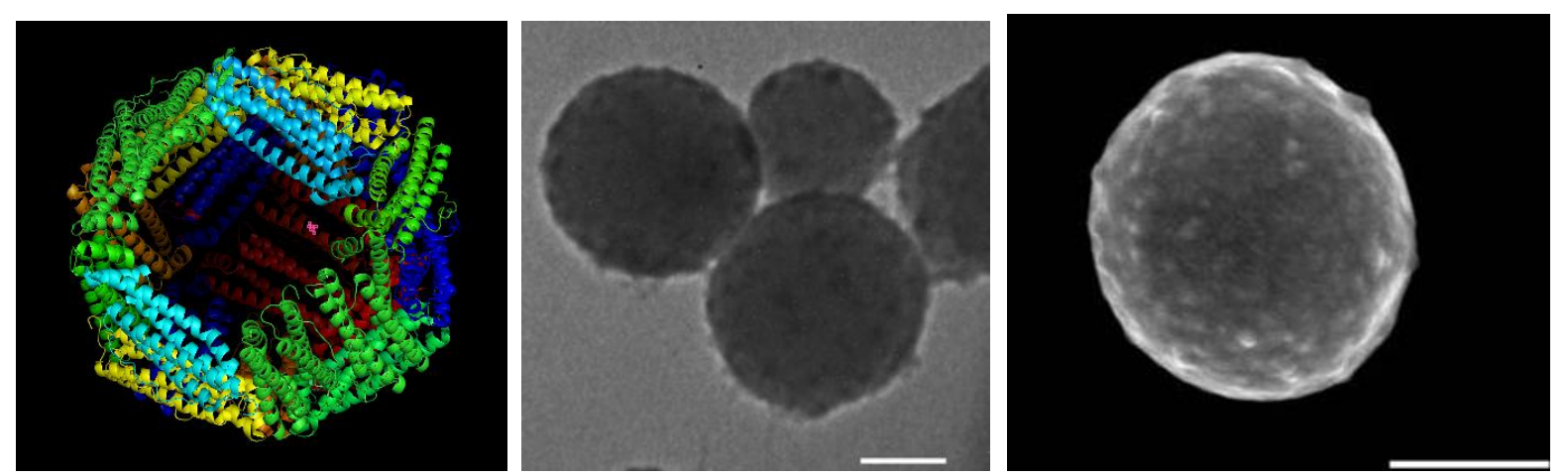

Figure 1 Ferritin protein chime structure (a), TEM (b) and FE-SEM images(c) of ferritin-P4VP (stained with uranyl acetate). Scale bars $=200 \mathrm{~nm}$.
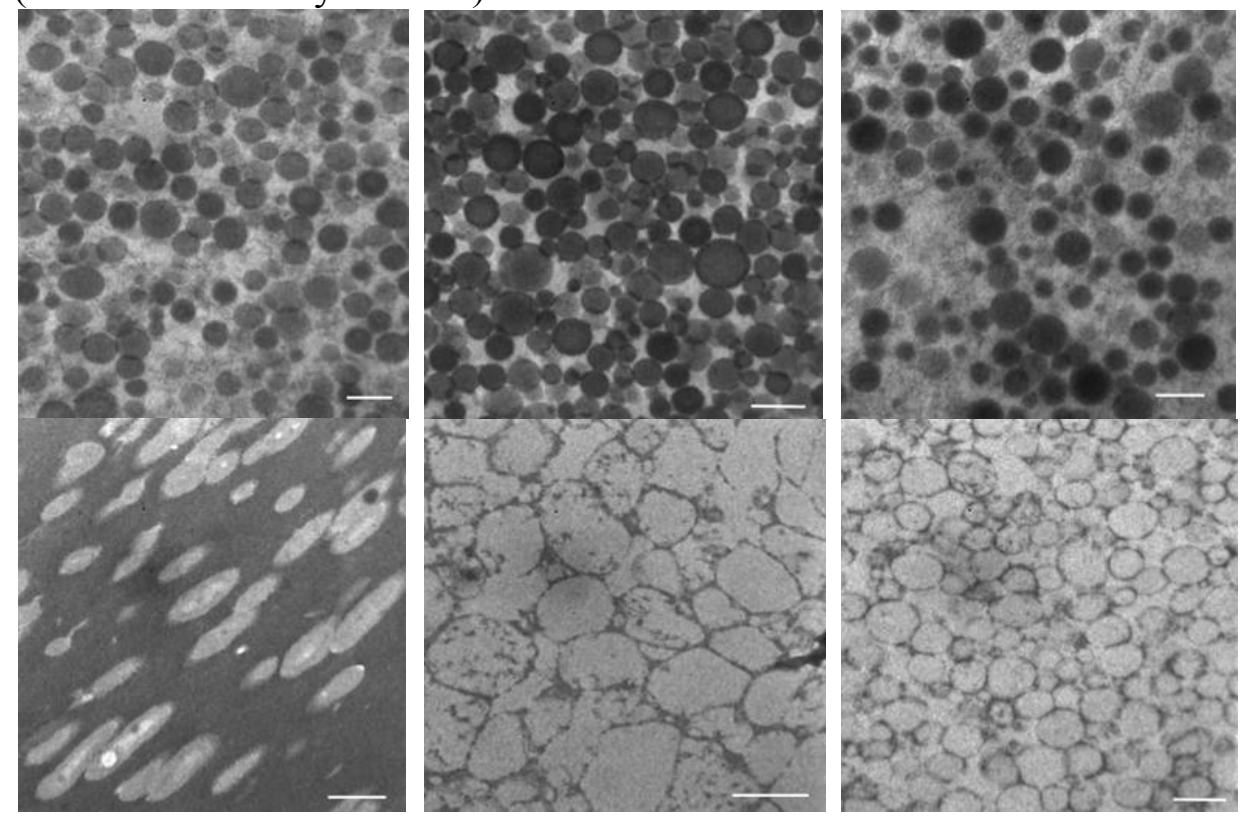

Figure 2 TEM images of ferritin-P4VP cross section after (a) conventional protocol: GTA $+\mathrm{OsO}_{4}+$ $\mathrm{EtOH}+\mathrm{A} / \mathrm{R}+\mathrm{Emb}$; (b) $\mathrm{GTA}+\mathrm{OsO}_{4}+\mathrm{EtOH}+\mathrm{Emb}$; (c) GTA + $\mathrm{OsO}_{4}+\mathrm{A} / \mathrm{R}+\mathrm{Emb}$; (d) GTA + $\mathrm{EtOH}+\mathrm{Emb}$; (e) GTA + Emb;(f) GTA + A/R + Emb. Scale bars $=500 \mathrm{~nm}$.

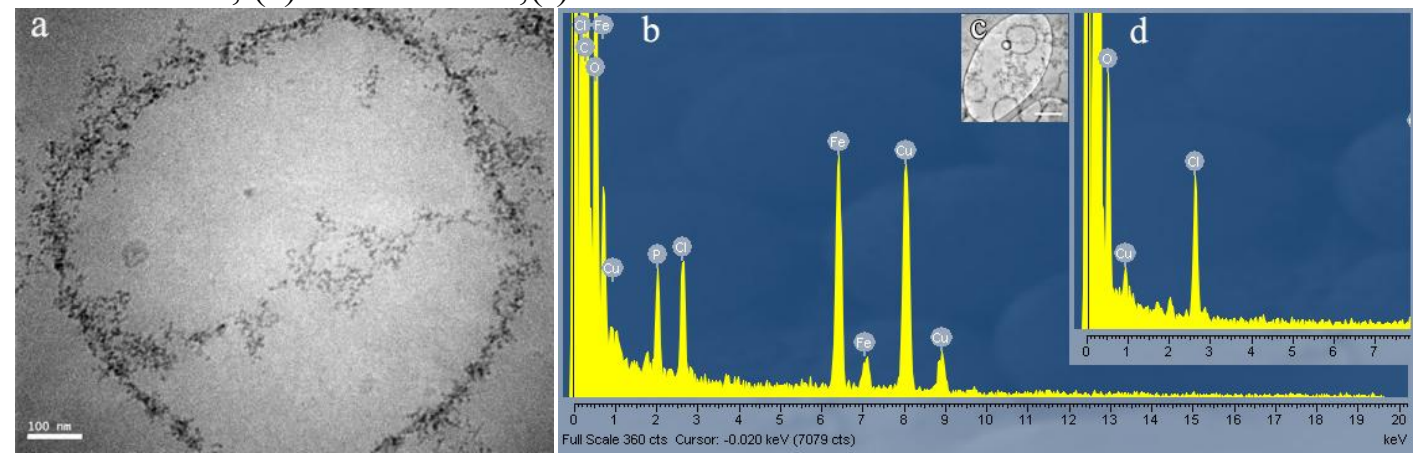

Figure 3 (a) HRTEM image of ferritin-P4VP tomography under optimal condition, (b, c) HRTEM EDS spectra and image of ferritin-P4VP hybrids, (d) HRTEM EDS spectra of embedding resin, as shown by arrows (Note the absence of $\mathrm{Fe}$ and $\mathrm{P}$ ), The $\mathrm{Cu}$ peaks in Fig $\mathrm{b}$ and $\mathrm{d}$ are from copper grid. 